\title{
Article \\ Influence of the Anthocyanin and Cofactor Structure on the Formation Efficiency of Naturally Derived Pyranoanthocyanins
}

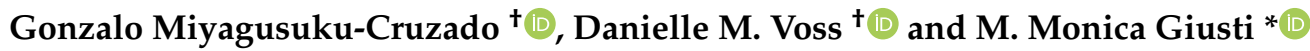 \\ Department of Food Science and Technology, The Ohio State University, 2015 Fyffe Rd., \\ Columbus, OH 43210 1007, USA; miyagusukucruzado.1@osu.edu (G.M.-C.); voss.129@osu.edu (D.M.V.) \\ * Correspondence: giusti.6@osu.edu; Tel.: +1-614-247-8016 \\ + These authors contributed equally to this work.
}

Citation: Miyagusuku-Cruzado, G.; Voss, D.M.; Giusti, M.M. Influence of the Anthocyanin and Cofactor Structure on the Formation Efficiency of Naturally Derived Pyranoanthocyanins. Int. J. Mol. Sci. 2021, 22, 6708. https://doi.org/ $10.3390 /$ ijms 22136708

Academic Editor: Maurizio Battino

Received: 14 May 2021

Accepted: 17 June 2021

Published: 23 June 2021

Publisher's Note: MDPI stays neutral with regard to jurisdictional claims in published maps and institutional affiliations.

Copyright: (c) 2021 by the authors. Licensee MDPI, Basel, Switzerland. This article is an open access article distributed under the terms and conditions of the Creative Commons Attribution (CC BY) license (https:/ / creativecommons.org/licenses/by/ $4.0 /)$.
Abstract: Pyranoanthocyanins are anthocyanin-derived pigments with higher stability to $\mathrm{pH}$ and storage. However, their slow formation and scarcity in nature hinder their industrial application. Pyranoanthocyanin formation can be accelerated by selecting anthocyanin substitutions, cofactor concentrations, and temperature. Limited information is available on the impacts of the chemical structure of the cofactor and anthocyanin; therefore, we evaluated their impacts on pyranoanthocyanin formation efficiency under conditions reported as favorable for the reaction. Different cofactors were evaluated including pyruvic acid, acetone, and hydroxycinnamic acids ( $p$-coumaric, caffeic, ferulic, and sinapic acid) by incubating them with anthocyanins in a molar ratio of 1:30 (anthocyanin:cofactor), $\mathrm{pH} 3.1$, and $45^{\circ} \mathrm{C}$. The impact of the anthocyanin aglycone was evaluated by incubating delphinidin, cyanidin, petunidin, or malvidin derivatives with the most efficient cofactor (caffeic acid) under identical conditions. Pigments were identified using UHPLC-PDA and tandem mass spectrometry, and pyranoanthocyanin formation was monitored for up to $72 \mathrm{~h}$. Pyranoanthocyanin yields were the highest with caffeic acid $(\sim 17 \%$ at $72 \mathrm{~h}, p<0.05)$. When comparing anthocyanins, malvidin-3-O-glycosides yielded twice as many pyranoanthocyanins after $24 \mathrm{~h}$ $(\sim 20 \%, p<0.01)$ as cyanidin-3-O-glycosides. Petunidin- and delphinidin-3-O-glycosides yielded $<2 \%$ pyranoanthocyanins. This study demonstrated the importance of anthocyanin and cofactor selection in pyranoanthocyanin production.

Keywords: hydroxyphenyl-pyranoanthocyanins; naturally derived pigments; accelerated formation; 10-catechyl-pyranoanthocyanins

\section{Introduction}

Anthocyanins (ACNs) are dietary flavonoids with bright colors that can range from red to blue [1,2]. The use of ACN-rich extracts as food colorants has increased in recent years due to potential behavioral concerns associated with the consumption of artificial dyes $[3,4]$. Also, interest in ACN consumption has grown due to their potential bioactive and healthpromoting properties [5]. However, from an industrial point of view, the application of ACN-rich extracts as food colorants is restricted due to limited long-term stability and color expression [1]. Additionally, common components in the food matrix such as ascorbic acid can bleach ACNs, resulting in their degradation [6]. Several mechanisms have been proposed for the stabilization of ACNs in foods such as copigmentation with phenolic compounds [7], complexation with proteins [8], encapsulation within polysaccharides [9], and chelation with metals [10].

Studies have shown that prolonged interaction of ACNs with hydroxycinnamic acids [7], acetone [11], or pyruvic acid (PA) [12] can result in the formation of ACN-derived pigments called pyranoanthocyanins (PACNs). This process occurs during winemaking as a result of the interaction between ACNs and yeast metabolites $[13,14]$. These ACN-derived pigments can express color across all $\mathrm{pH}$ values [11] and have better long-term storage 
stability $[6,11]$. Moreover, PACNs showed enhanced resistance to bleaching by ascorbic acid [6] and sulfur dioxide [11,15]. These pigments are formed through the cycloaddition reaction of a reactive cofactor on position $\mathrm{C} 4$ and the $5-\mathrm{OH}$ group of the $\mathrm{ACN}$ molecule, resulting in the formation of an additional pyran ring [16] and consequently, in the unavailability of $C 4$ to participate in degradation reactions [17]. Studies on the synthesis pathways of different PACNs from ACN-rich extracts have shown that carboxy-PACNs can result from the reaction with PA, hydroxyphenyl-PACNs from the reaction with hydroxycinnamic acids, and methyl-PACNs from the reaction with acetone [11,18]. Due to this formation process, ACNs with substitutions on position $\mathrm{C} 5$ cannot form $\mathrm{PACNs}$, thus excluding many pigment sources commonly used in the food industry from being PACN precursors. ACN sources commonly used in the food industry capable of forming PACNs include black carrot [7,19], elderberries [7], and some grape extracts [20].

Although more stable, PACNs are hard to find in nature with limited quantities reported in onions [21] and strawberries [22]. Additionally, PACNs can be found in a small number of foods such as wine [18], sumac [23], fruit wines [24], and fruit juices after extended periods of time [19]. Most studies have focused on the occurrence and identification of PACNs $[18-20,25]$, their stability $[6,11]$, and their unique color characteristics [26]. However, little has been reported on the optimization of their formation. Previous studies reported that PACN formation efficiency is increased with 1-6 di-glycosylated ACNs [12], the removal of ACN aromatic acylating groups [27], solution $\mathrm{pH} \sim 3.0$ [28], as well as higher incubation temperatures [29] and molar cofactor ratios [28,29]. In addition, studies comparing different cofactors showed greater PACN yields with caffeic acid (CA) than with PA [27]. However, the impact on PACN formation efficiency of other cofactors, such as acetone, and modifications dependent on cofactor type and ACN structure are still underreported. This information is important if PACNs are to be used by the food industry as it would help in the development of more stable, naturally derived colorants. Therefore, the objective of this study was to determine the effect of the chemical structure of the cofactor and ACN on PACN formation efficiency under accelerated conditions ( $\mathrm{pH}$ 3.1, $\mathrm{ACN}$ to cofactor molar ratio of 1:30, incubation at $45^{\circ} \mathrm{C}$ ).

\section{Results}

\subsection{Evaluation of Pyranoanthocyanin Formation Efficiency Using Different Types of Cofactors}

$\mathrm{CA}$, acetone, and PA were tested as cofactors for the formation of PACNs from cyanidin-glycosides obtained from saponified black carrot ACN extracts (sBC) with results shown in Figure 1. The ACN extract was mainly composed of cyanidin-3-O-xylosylglucosyl-galactoside (peak 1, C3XyGlGa, $\sim 64 \%$ of total area at $475-520 \mathrm{~nm}$ ) and cyanidin-3$O$-xylosyl-galactoside (peak 2, C3XyGa, $\sim 34 \%$ of total area at $475-520 \mathrm{~nm}$ ). The $475-520 \mathrm{~nm}$ max plot chromatogram was used because it accounted for the $\lambda_{\text {vis-max }}$ of all pigments evaluated in this study. After incubation with CA, two new peaks were detected in the PDA chromatogram. Their hypsochromic shift of $\lambda_{\text {vis-max }}$ compared to the precursor ACNs, later retention times, and MS spectra revealed that these new peaks corresponded to 10catechyl-PACNs derived from the ACNs previously identified in sBC. Consequently, peak 3 was ascribed to 10-catechyl-pyranocyanidin-3-O-xylosyl-glucosyl-galactoside and peak 4 to 10 -catechyl-pyranocyanidin-3-O-xylosyl-galactoside. No new peaks were detected in the cofactor-free control and samples with acetone and PA after $72 \mathrm{~h}$ of incubation. 


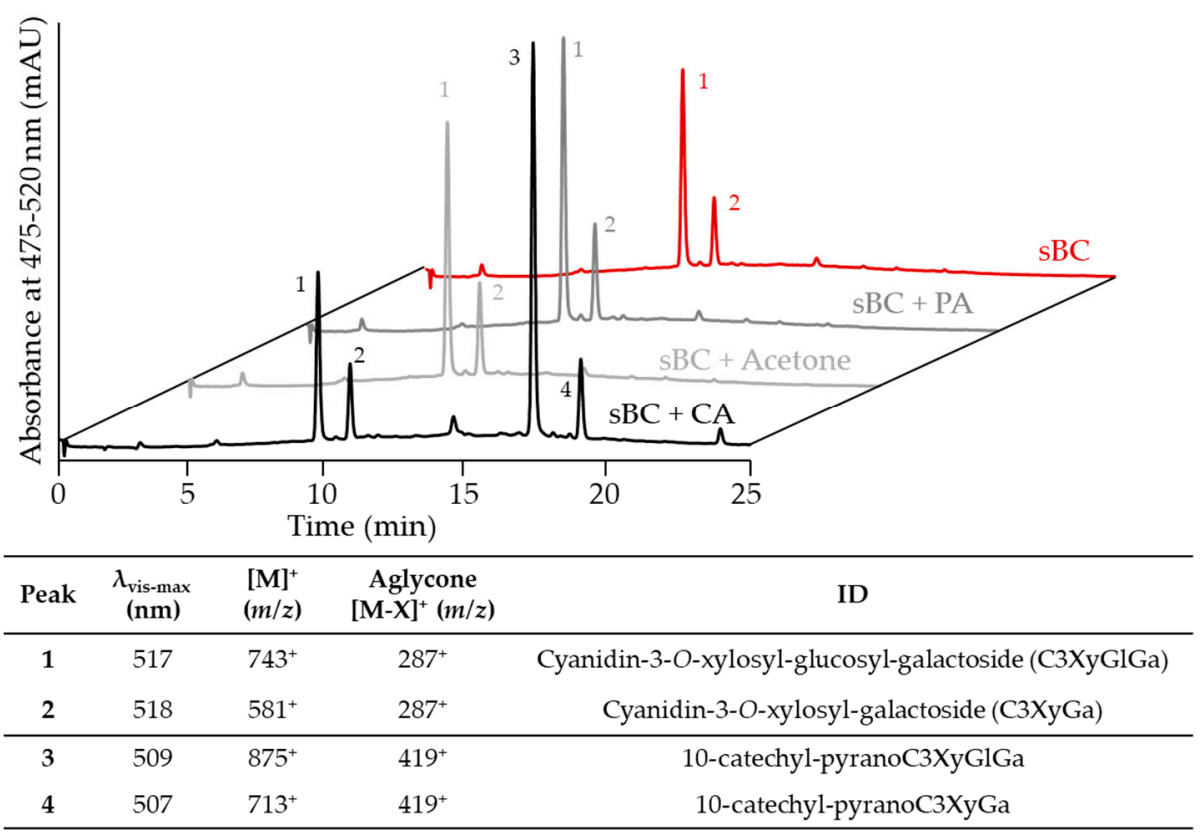

Figure 1. Comparing the PACN formation after $72 \mathrm{~h}$ of anthocyanin (saponified black carrot, $\mathrm{sBC}$ ) incubation with caffeic acid (CA), acetone, or pyruvic acid (PA). Chromatograms show the max plot in the 475-520 $\mathrm{nm}$ range. Table shows the wavelength of maximum absorption in the visible range $\left(\lambda_{\text {vis-max }}\right)$, mass per charge ratio $(m / z)$ of the main ion and its aglycone, and tentative identity (ID).

Yields of PACNs, calculated as percentages compared to the initial ACN content using Equation (1), increased from $3.9 \pm 0.2 \%$ at $24 \mathrm{~h}$ to $14.1 \pm 0.5 \%$ and $17.2 \pm 0.6 \%$ after 48 and $72 \mathrm{~h}$ of incubation with CA at $45^{\circ} \mathrm{C}$, respectively (Figure 2). Based on the peak areas calculated at each time point using Equation (3), PACNs comprised 4.6 $\pm 0.2,38.4 \pm 3.5$, and $63.5 \pm 4.2 \%$ of the total pigment at 24,48 , and $72 \mathrm{~h}$, respectively. No PACNs were detected in samples incubated with acetone or PA. The pigment remaining after $72 \mathrm{~h}$ of incubation was the highest in the samples with CA $(27.4 \pm 2.6 \%)$. This content was higher than the one observed in samples with PA $(16.4 \pm 1.8 \%)$, but not significantly so $(p=0.06)$. Additionally, the pigment remaining in samples with CA was significantly higher than the ones observed in cofactor-free control samples $(9.1 \pm 1.9 \%, p<0.01)$ and samples with acetone $(12.1 \pm 2.8 \%, p<0.01)$.

PACN yields calculated using Eq. 1 when using $\mathrm{CA}$ as cofactor

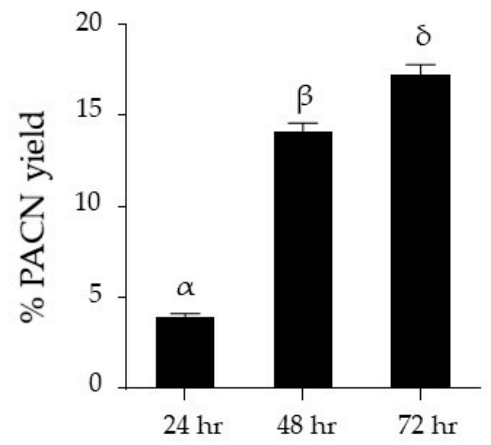

Pigment remaining calculated using Eq. 2 after incubation with CA, acetone, or PA

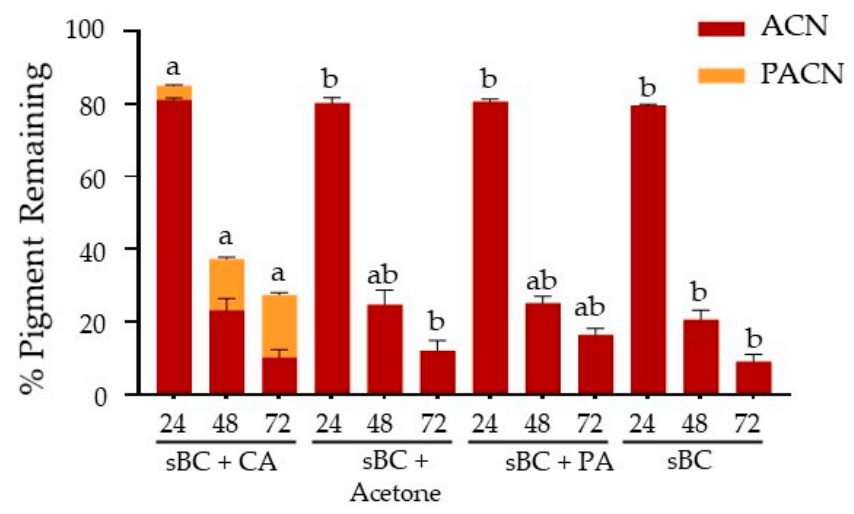

Figure 2. Pyranoanthocyanin yield (\%PACN) with caffeic acid (CA) and pigment remaining (\%) after incubation with different cofactors for 24,48 , and $72 \mathrm{~h}$ at $45{ }^{\circ} \mathrm{C}$ with cyanidin-glycosides (from saponified black carrot, sBC). Different Greek letters show significant differences among time points at a 0.05 level. Different letters show statistically significant differences among cofactors at the same time point at a 0.05 level. Results are expressed as means \pm standard error $(n=3)$. ACN: anthocyanin, PA: pyruvic acid. 


\subsection{Evaluation of Pyranoanthocyanin Formation Efficiency Using Different Hydroxycinnamic Acids}

After incubation for up to $72 \mathrm{~h}, \mathrm{CA}$ was the only cofactor forming PACN under our experimental conditions, strongly suggesting that the chemical structure of the cofactor can impact PACN yields. Studies have shown different reactivity rates of hydroxycinnamic acids with ACNs or PACNs resulting in the formation of new pigments [30,31]. In these studies, it was hypothesized that the nucleophilic nature of the hydroxycinnamic acid impacted its reactivity [31], with di- and trisubstituted ones more reactive than monosubstituted ones [30]. Therefore, we hypothesized that minor structural differences among hydroxycinnamic acids (CA, $p$-coumaric acid (pCA), ferulic acid (FA), and sinapic acid (SA)) may also affect PACN yields under accelerated conditions. Analysis with UHPLC-PDA coupled to a tandem mass spectrometer with electrospray ionization (ESI-MS/MS) showed that the identities of peaks 1-4 (deriving from incubation with CA) in Figure 3 were consistent with the ones previously reported in Figure 1. The incubation with the other hydroxycinnamic acids resulted in the formation of new compounds denoted by peaks 5-10 with later retention times and hypsochromic effects on $\lambda_{\text {vis-max }}$ (Figure 3). These characteristics along with the $[\mathrm{M}]^{+}$and $[\mathrm{M}-\mathrm{X}]^{+}$values were consistent with hydroxyphenyl-PACNs derived from non-acylated ACNs present in sBC. Peaks 5 and 6 were attributed to 10-phenyl-pyranocyanidin-3-O-xylosylglucosyl-galactoside and 10-phenyl-pyranocyanidin-3-O-xylosyl-galactoside, respectively. Similarly, peaks 7 and 8 were recognized as 10-guaiacyl derivatives and peaks 9 and 10 as 10-syringyl derivatives (Figure 3).

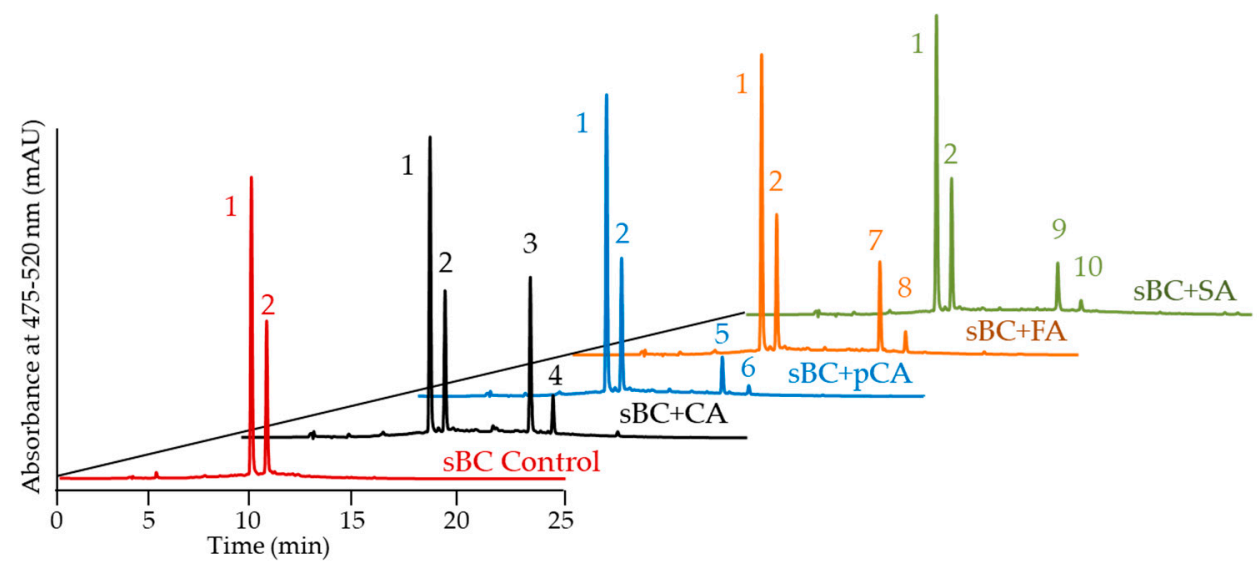

\begin{tabular}{|c|c|c|c|c|c|c|c|c|c|}
\hline Peak & $\begin{array}{c}\lambda_{\text {vis-max }} \\
(\mathbf{n m})\end{array}$ & $\begin{array}{l}{[\mathrm{M}]^{+}} \\
(m / z)\end{array}$ & $\begin{array}{c}\text { Aglycone } \\
{[\mathrm{M}-\mathrm{X}]^{+}(\mathrm{m} / \mathrm{z})}\end{array}$ & ID & Peak & $\begin{array}{c}\lambda_{\text {vis-max }} \\
\text { (nm) }\end{array}$ & $\begin{array}{l}{[\mathrm{M}]^{+}} \\
(m / z)\end{array}$ & $\begin{array}{c}\text { Aglycone } \\
{[\mathrm{M}-\mathrm{X}]^{+}(m / z)}\end{array}$ & ID \\
\hline 1 & 517 & $743^{+}$ & $287^{+}$ & C3XyGlGa & 6 & 508 & $697^{+}$ & $403^{+}$ & 10-phenyl-pyranoC $3 \mathrm{XyGa}$ \\
\hline 2 & 518 & $581^{+}$ & $287^{+}$ & C3XyGa & 7 & 503 & $873^{+}$ & $417^{+}$ & 10-guaiacyl-pyranoC3XyGlGa \\
\hline 3 & 509 & $875^{+}$ & $419^{+}$ & 10-catechyl-pyranoC3XyGlGa & 8 & 501 & $711^{+}$ & $417^{+}$ & 10-guaiacyl-pyranoC3XyGa \\
\hline 4 & 507 & $713^{+}$ & $419^{+}$ & 10-catechyl-pyranoC3XyGa & 9 & 513 & $887^{+}$ & $431^{+}$ & 10-syringyl-pyranoC3XyGlGa \\
\hline 5 & 509 & $859^{+}$ & $403^{+}$ & 10-phenyl-pyranoC 3 XyGlGa & 10 & 512 & $725^{+}$ & $431^{+}$ & 10-syringyl-pyranoC3XyGa \\
\hline
\end{tabular}

Figure 3. Comparing the pyranoanthocyanin formation after $72 \mathrm{~h}$ of anthocyanin (saponified black carrot, sBC) incubation with caffeic acid (CA), $p$-coumaric acid (pCA), ferulic acid (FA), and sinapic acid (SA). Chromatograms show the max plot in the $475-520 \mathrm{~nm}$ range. Table shows the wavelength of maximum absorption in the visible range $\left(\lambda_{\text {vis-max }}\right)$, mass-per-charge ratio $(m / z)$ of the main ion and its aglycone, and tentative identity (ID). C3XyGlGa: cyanidin-3-O-xylosyl-glucosylgalactoside, C3XyGa: cyanidin-3-O-xylosyl-galactoside.

Yields calculated using Equation (1) and displayed in Figure 4 showed that after $24 \mathrm{~h}$ of incubation, PACN yield with CA $(12.5 \pm 0.2 \%)$ was significantly greater than with FA $(8.3 \pm 0.2 \%, p<0.01)$. Moreover, FA had significantly higher yields than $\mathrm{pCA}(3.5 \pm 0.3 \%$, $p<0.01)$ and SA $(4.0 \pm 0.2 \%, p<0.01)$, with no significant differences among these last two cofactors $(p>0.05)$. After $48 \mathrm{~h}$ of incubation, the same efficiency pattern was observed. After $72 \mathrm{~h}$ of incubation, CA and FA were significantly more efficient than pCA ( $p=0.0476$ and $p=0.0246$, respectively). Yields with SA were lower than with CA and FA, but not 
significantly so $(p>0.05$ for both CA and FA). When analyzing the percent of pigment remaining, results in Figure 4 showed that after $24 \mathrm{~h}$, incubation with FA $(41.2 \pm 2.6 \%)$ or SA $(35.8 \pm 3.2 \%)$ resulted in a significantly lower pigment remaining $(p=0.0367$ and $p=0.3350$, respectively) compared to the cofactor-free control $(55.8 \pm 1.4 \%)$. However, these differences were not significant after $72 \mathrm{~h}$ of incubation $(p>0.05)$.

PACN yields calculated using Eq. 1 when using different hydroxycinnamic acids as cofactors

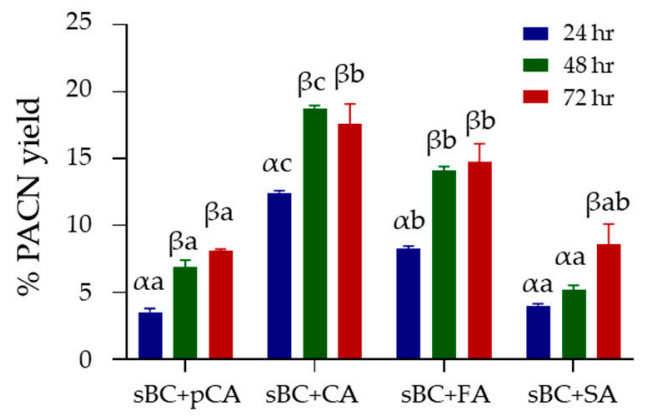

Pigment remaining calculated using Eq. 2 after incubation with different hydroxycinnamic acids

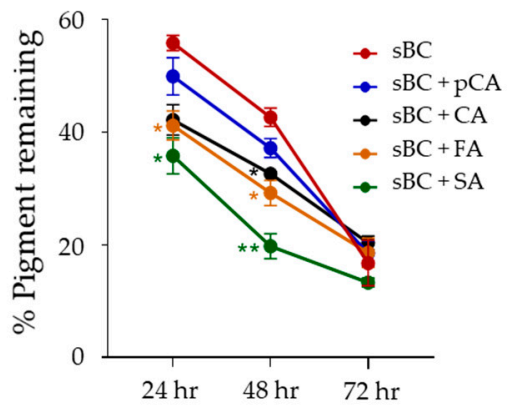

Figure 4. Pyranoanthocyanin yield (\%PACN) with different hydroxycinnamic acids and pigment remaining (\%) after incubation for 24,48 , and $72 \mathrm{~h}$ at $45^{\circ} \mathrm{C}$ with cyanidin-glycosides (from saponified black carrot, sBC). Different Greek letters show significant differences among time points at a 0.05 level. Different letters show significant differences among cofactors at the same time point at a 0.05 level. Asterisks $\left({ }^{*}\right)$ and $\left({ }^{* *}\right)$ indicate significant differences against the cofactor-free control (sBC) at a 0.05 and 0.01 level, respectively. Results are expressed as means \pm standard error $(n=3)$. pCA: $p$-coumaric acid. CA: caffeic acid, FA: ferulic acid, SA: sinapic acid, Eq: equation.

\subsection{Evaluation of Pyranoanthocyanin Formation Efficiency Using Different Anthocyanins}

Minor structural differences among hydroxycinnamic acids impacted PACN yields. Therefore, we hypothesized that minor structural differences among ACNs could also impact PACN yields. Berberis boliviana was chosen as the pigment source for this comparison because it contains simple ACNs deriving from four different aglycones in similar proportions [32]. Results confirmed the presence of delphinidin, cyanidin, petunidin, and malvidin derivatives in the Berberis boliviana $\mathrm{ACN}$ extract used in this study. In addition, results showed the presence of two glycosylation patterns consistent with glucoside, denoted by a neutral loss of $162 \mathrm{~m} / z$, and rutinoside, denoted by a neutral loss of $308 \mathrm{~m} / z$ (Figure 5). After fractionation using semi-preparative HPLC-PDA, four fractions were obtained each with glucoside and rutinoside derivatives of different ACN aglycones. Further UHPLC-PDA-ESI-MS/MS analyses were carried out to identify the individual ACNs in each fraction, and their identities were compared against previous literature. The pigments in the delphinidin-derivatives fraction $(\mathrm{Dp})$ were delphinidin3-O-glucoside (Dp1, 91\%) and delphinidin-3-O-rutinoside (Dp2, 7\%), pigments in the cyanidin-derivatives fraction $(\mathrm{Cy})$ were cyanidin-3-O-glucoside $(\mathrm{Cy} 1, \sim 65 \%)$ and cyanidin3-O-rutinoside ( $\mathrm{Cy} 2, \sim 27 \%$ ) with a minor content of petunidin-3-O-glucoside (Pt1, $8 \%$ ), pigments in the petunidin-derivatives fraction $(\mathrm{Pt})$ were petunidin-3-O-glucoside (Pt1, $\sim 73 \%$ ) and petunidin-3-O-rutinoside (Pt2, 27\%), and pigments in the malvidin-derivatives fraction (Mv) were malvidin-3-O-glucoside (Mv1, $77 \%$ ) and malvidin-3-O-rutinoside (Mv2, 22\%). 


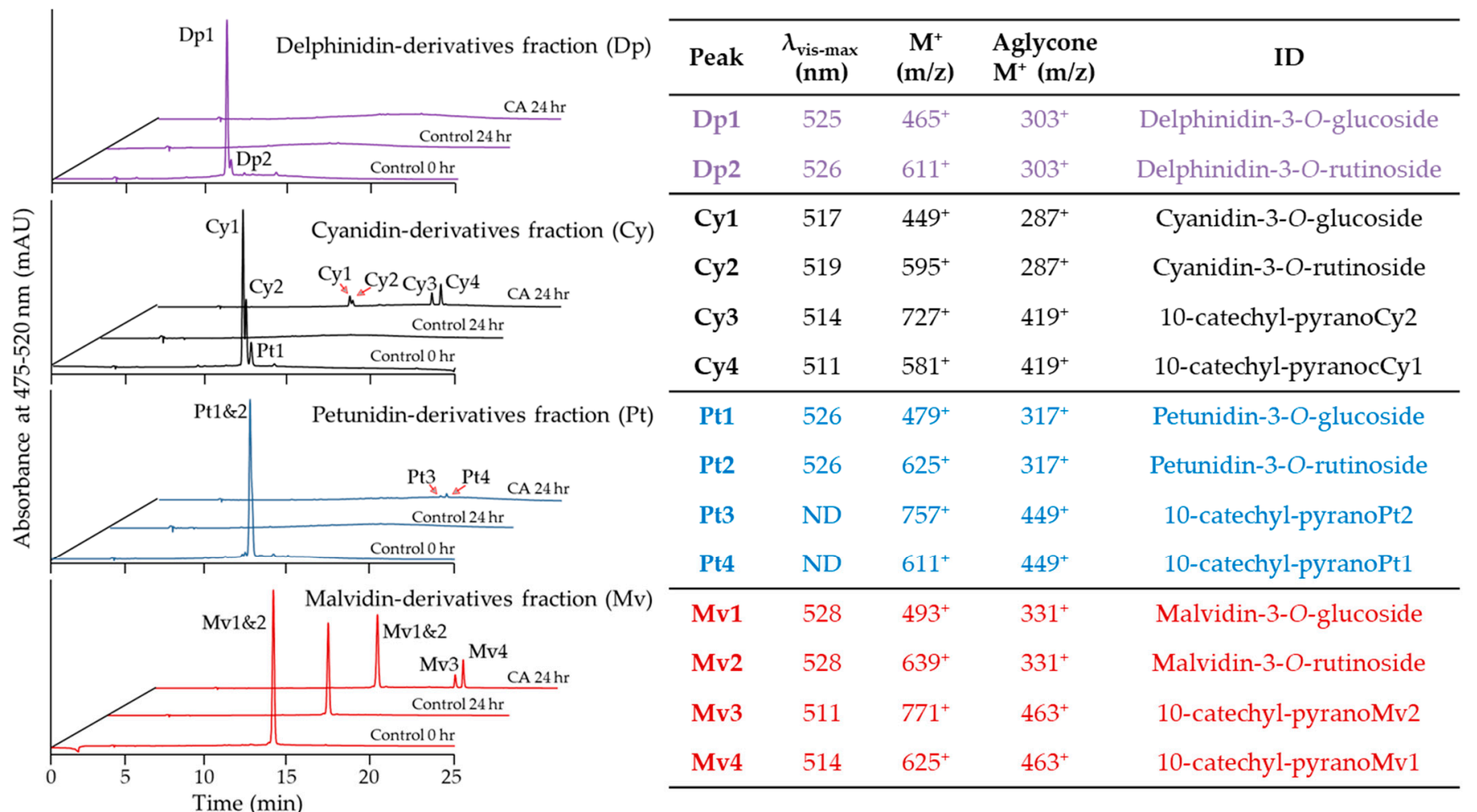

Figure 5. Anthocyanins from fractions with different aglycones and PACN formation after $24 \mathrm{~h}$ of $45^{\circ} \mathrm{C}$ incubation with caffeic acid (CA). Chromatograms show the max plot in the 475-520 nm range. Table shows the wavelength of maximum absorption in the visible range $\left(\lambda_{\text {vis-max }}\right)$, mass-per-charge ratio $(m / z)$ of the main ion and its aglycone, and tentative identity (ID).

After $24 \mathrm{~h}$ incubation with CA, fractions with cyanidin-, petunidin-, or malvidinderived ACNs developed peaks with later retention times and hypsochromic $\lambda_{\text {vis-max }}$. UHPLC-PDA-ESI-MS/MS analyses showed that these new peaks were consistent with 10-catechyl derivatives of the ACNs present in each fraction. Indeed, peaks $\mathrm{Cy} 3$ and Cy4 were named 10-catechyl-pyranocyanidin derivatives, peaks Pt3 and Pt4 10-catechylpyranopetunidin derivatives, and peaks Mv3 and Mv4 10-catechyl-pyranomalvidin derivatives (Figure 5). It is worth noting that incubation of delphinidin-derivatives with CA yielded no PACNs under our experimental conditions.

The highest PACN yield (Figure 6) was observed for malvidin-derivatives followed by cyanidin-derivatives $(19.8 \pm 1.2 \%$ and $9.2 \pm 0.8 \%$, respectively, after $24 \mathrm{~h}$ of incubation, $p<0.01$ ). Furthermore, using malvidin-derivatives not only yielded more PACNs but also resulted in a significantly higher percent of pigment remaining after incubation $(64.1 \pm 5.2 \%, p<0.01)$, followed by cyanidin-derivatives $(16.0 \pm 2.2 \%)$ and then petunidinderivatives $(1.9 \pm 0.1 \%)$.

When analyzing the relative content of PACNs with a rutinoside substitution as a percentage of total PACNs (Figure 5), this content was $36.1 \pm 0.2 \%$ in the cyanidin-derivatives fraction, $32.4 \pm 1.1 \%$ in the petunidin-derivatives fraction, and $31.4 \pm 0.4 \%$ in the malvidinderivatives fraction. These values were higher than the ACN-3-O-rutinoside proportion in the original ACN extract analyzed in our preliminary analysis (data not shown). 
PACN yields calculated using Eq. 1 when using fractions with different anthocyanin aglycones with CA

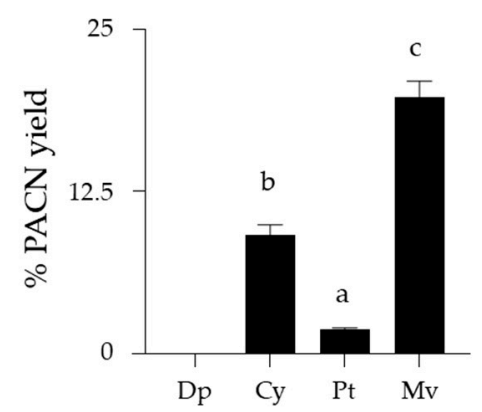

Pigment remaining calculated using Eq. 2 after incubation of fractions with different anthocyanin aglycones with CA

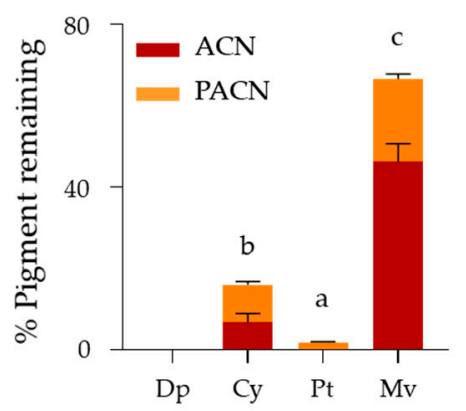

Figure 6. Pyranoanthocyanin yield (\%PACN) and pigment remaining (\%) after incubation of anthocyanin fractions with different aglycones with caffeic acid (CA) for $24 \mathrm{~h}$ at $45^{\circ} \mathrm{C}$. Different letters show significant differences among different fractions at a 0.05 level. Results are expressed as mean \pm standard error $(n=3)$. ACN: anthocyanin, Dp: delphinidin-derivatives fraction, Cy: cyanidin-derivatives fraction, Pt: petunidin-derivatives fractions, Mv: malvidin-derivatives fraction, Eq: equation.

\section{Discussion}

Under accelerated formation conditions (pH 3.1, 1:30 ACN to cofactor molar ratio, and $45{ }^{\circ} \mathrm{C}$ incubation temperature), PACNs were detected in as little as $24 \mathrm{~h}$ with yields up to $19.8 \%$ using malvidin-derived ACNs and CA as the cofactor. These yields were at least six times higher than those reported for malvidin-3-O-glucoside with $\mathrm{CA}$ after four months using wine-like systems ( $\mathrm{pH} 3.2, \sim 1: 2 \mathrm{ACN}$ to cofactor molar ratio, and incubation at $15^{\circ} \mathrm{C}$ ) [31]. Additionally, the PACN yields obtained under our experimental conditions were at least five times higher than those reported for malvidin-3-O-glucoside with CA or FA and 20 times higher than those reported with pCA in synthetic grape mediums after 14 days ( $\mathrm{pH} 3.5, \sim 1: 0.8 \mathrm{ACN}$-to-cofactor molar ratio, and incubation at $30^{\circ} \mathrm{C}$ ) [33]. The higher yields observed in our study could be attributed to the use of conditions previously reported to accelerate PACN formation [27-29]. However, our results demonstrated that other factors such as the cofactor chemical structure and the ACN type also played a key role in PACN formation efficiency under accelerated formation conditions.

The PACN yields observed in this study using cyanidin-derived ACNs and CA were comparable to previous reports evaluating yields under similar conditions. Straathof and Giusti reported PACN yields of $\sim 20 \%$ after $24 \mathrm{~h}$ of incubation at $65{ }^{\circ} \mathrm{C}$ using elderberry ACNs with CA [29], which was higher than those obtained under our experimental conditions when comparing different hydroxycinnamic acids as cofactors. This higher yield could be attributed to higher incubation temperatures and molar cofactor ratio used (1:50) as well as the different glycosylation patterns of elderberry ACNs. To evaluate potential differences in PACN yields as a result of the use of different cofactors and $\mathrm{ACN}$ types, $45^{\circ} \mathrm{C}$ instead of $65^{\circ} \mathrm{C}$ was selected to reduce pigment degradation as reported by Straathof and Giusti [29] at temperatures of $65^{\circ} \mathrm{C}$ or above. In addition, our PACN yields after $72 \mathrm{~h}$ of incubation were similar to the ones reported by Zhu and Giusti after seven days of incubation at $25^{\circ} \mathrm{C}$ [27]. In that study, incubation for seven additional weeks led to higher PACN yields ( $71-85 \%)$ that were not obtained under the accelerated conditions used in our experiment.

To compare different cofactors, black carrot was selected as a pigment source because its ACNs do not have glycosylations on position C5 [7], and this allowed for the cycloaddition reaction with a reactive adduct resulting in the formation of a pyran ring between the $\mathrm{C} 4$ and 5-OH groups of the ACN molecule [16]. Moreover, the tri- and di-glycosylation patterns at $\mathrm{C} 3$ of these ACNs allowed for better solubility of their derived PACNs, preventing their precipitation as observed in our preliminary studies with ACNs from other sources. 
As sinapoyl acylating groups have been shown to decrease PACN formation efficiency [27], alkaline hydrolysis was carried out to remove the aromatic acylating moiety.

Among the cofactors tested (CA, acetone, and PA), CA was the only one that produced PACNs under our experimental conditions. Cofactors with an aromatic ring, such as hydroxycinnamic acids, can copigment with ACNs [7,34]. Through intermolecular interactions, the aromatic cofactor is brought in closer proximity to the $\mathrm{ACN}$, which can stabilize the chromophore during the incubation period and increase the likelihood of a reaction $[27,28,35]$. Copigments must contain a benzene ring for $\pi-\pi$ interactions with ACNs to take place [36]. Aliphatic cofactors such as PA and acetone are not expected to copigment with ACNs, which could reduce their interaction with ACNs thus resulting in a lower PACN yield compared to CA [27]. Previous studies have found PACN formation yields 3.5 to 4 times greater with CA as a cofactor than with PA $[27,28]$. While we did not detect PACNs forming from PA even after $72 \mathrm{~h}$, the longer incubation periods of 42 and 56 days and greater molar ratios of PA (1:100 and 1:200) used in those studies may have compensated for the less efficient cofactor-ACN interaction to facilitate PACN formation $[27,28]$.

CA was the most efficient cofactor even when compared to other hydroxycinnamic acids, yielding the highest amount of PACNs after only $24 \mathrm{~h}$ of incubation $(\mathrm{CA}>\mathrm{FA}>\mathrm{SA}=\mathrm{pCA})$. However, after $72 \mathrm{~h}$, no statistical differences were observed in the PACN yield between samples incubated with CA, FA, or SA, although yields with SA were lower and not statistically different than yields with pCA. CA and FA are widely distributed in natural sources. CA is abundant in plants such as aronia berries [37] and black carrots [38], and FA is found in plant cell walls and can be released into a solution by alkaline hydrolysis [39]. Therefore, the saponification process we used to remove the acylating group in the ACN molecule making it more predisposed for PACN formation [27] can, at the same time, release one of the most efficient cofactors into a solution.

The efficiency pattern of hydroxycinnamic acids observed in our results is similar to the one reported in wine-like model solutions [31,40], despite differences in cofactor molar ratios, incubation temperatures, and $\mathrm{PACN}$ yields. Additionally, similar reactivity patterns were observed for hydroxycinnamic acids during the formation of portisins [30]. It has been hypothesized that bond formation between $\mathrm{C} 4$ of the ACN and the alpha carbon of the hydroxycinnamic acid is affected by the nucleophilic nature of the cofactor [40]. The stronger nucleophilic nature of CA as a result of the two hydroxyl substitutions in the phenolic ring [41] could explain its higher PACN formation efficiency. Additionally, steric hindrance impacts PACN formation efficiency $[12,26]$; this hindrance may explain the higher PACN yields with FA over SA as cofactors.

When evaluating the impact of the ACN aglycone on PACN formation using CA as a cofactor, malvidin was the most efficient followed by cyanidin (malvidin > cyanidin > petunidin). Malvidin-3-O-glucoside has been shown to have stronger copigmentation interactions with CA than other ACN-3-O-glucosides [34]. As copigmentation with the cofactor may facilitate PACN formation [27], the increased interactions may contribute to greater PACN formation rates with malvidin-derived ACNs. This stronger copigmentation with CA may also help to explain the significantly higher pigment remaining after incubation for $24 \mathrm{~h}$. Additionally, the higher content of ACNs remaining in the malvidin fraction after $24 \mathrm{~h}$ incubation ( $45 \%$ of initial pigment) may indicate that greater PACN yields may be achieved with longer incubation times. Results also showed very small amounts of PACNs in samples with petunidin-derived pigments $(\sim 1.9 \%)$ and no PACNs detected in samples with delphinidin-derivatives. Furthermore, after incubation at $45^{\circ} \mathrm{C}$ for $24 \mathrm{~h}$, there was little to no pigment remaining in these samples; this negates the possibility of PACN formation with increased incubation times. Delphinidin's and petunidin's propensity to degrade during prolonged heating $[42,43]$ may explain the absence or reduced formation of PACNs in these samples. The significantly higher yields of malvidin- and cyanidin-derived PACNs may explain why most reports have especially identified these derivatives $[11,14,16,19,25,44,45]$. 
The ACN fractions used to compare the impact of the aglycone type contained two glycosylation patterns (glucoside and rutinoside). Glycosylation patterns have been reported to impact PACN yields with cyanidin-3-O-rutinoside yielding more PACN than cyanidin-3-O-glucoside when incubated with PA at $25^{\circ} \mathrm{C}$ [12]. This observation was consistent with our results; indeed ACN-3-O-rutinosides seemed to form PACNs more efficiently than their glucoside counterparts denoted by an increased content of rutinoside-derivatives in the PACN fraction after incubation with CA (from $27 \%$ to $~ 37 \%$ in the Cy fraction, from $\sim 27 \%$ to $\sim 33 \%$ in the $\mathrm{Pt}$ fraction, and from $\sim 22 \%$ to $\sim 32 \%$ in the Mv fraction). Despite having similar glucoside to rutinoside proportions, $\mathrm{PACN}$ yields with $\mathrm{CA}$ were significantly different between the $\mathrm{Cy}, \mathrm{Pt}$, and $\mathrm{Mv}$ fractions. This difference suggests that the impact of the glycosylation may be secondary to the impact of the aglycone type on PACN yield, possibly due to the aglycone influence on stability and copigmentation ability.

\section{Materials and Methods}

\subsection{Plant Material, Chemical, and Reagents}

Berberis boliviana freeze-dried berries were generously donated by Carla del Carpio from Universidad Nacional de San Antonio Abad del Cusco (UNSAAC, Cusco, Peru). A commercial black carrot color powder (Daucus carota) was provided by D.D. Williamson (Louisville, KY, USA). CA, FA, SA, and PA were purchased from Sigma-Aldrich (St. Louis, MO, USA). Acetone, chloroform, acetonitrile, UHPLC-grade water, pCA, sodium benzoate, and sodium hydroxide were obtained from Fisher Scientific (Pittsburgh, PA, USA). Potassium sorbate was obtained from Spectrum (New Brunswick, NJ, USA). All other reagents and solvents were of at least analytical or HPLC grade unless otherwise indicated.

\subsection{Anthocyanin Preparation}

ACN extraction was carried out following the methodology described by RodriguezSaona and Wrolstad [46] with minor modifications. Black carrot color powders were prepared for extraction by reconstituting them in water. Berberis boliviana berries were deseeded manually, soaked in water, and frozen with liquid nitrogen before extraction. Then, the frozen berries and reconstituted black carrot color powder were each blended with $0.01 \% \mathrm{HCl}$ acetone $(v / v)$ using a Waring laboratory blender. The slurry was filtered through an $\mathrm{N}^{\circ} 4$ Whatman filter paper, and the cake was re-extracted using $0.01 \% \mathrm{HCl}$ aqueous acetone $(70 \% v / v)$ until a faint pink solution was obtained. The filtrate was then partitioned using chloroform for a final acetone:chloroform proportion of 1:2 $(v / v)$ in a separatory funnel and stored overnight at $4{ }^{\circ} \mathrm{C}$. The upper aqueous layer containing ACNs and other phenolic compounds was collected, and residual solvents were evaporated using a Büchi rotavapor at $45^{\circ} \mathrm{C}$ (Büchi, Flawil, Switzerland).

\subsection{Saponification of Black Carrot Anthocyanins}

Alkaline hydrolysis of acylated ACNs from black carrot was carried out to remove the acylation moiety in their structure following the methodology described by Giusti and Wrolstad [47]. Briefly, solutions rich in black carrot ACNs were mixed with $10 \% \mathrm{KOH}(w / v)$ in a proportion of 1:10 and left to stand for $8 \mathrm{~min}$ in the dark at room temperature, after which the $\mathrm{pH}$ was neutralized using $2 \mathrm{~N} \mathrm{HCl}$. Neutralized, saponified extracts were subjected to solid phase extraction (SPE) following the methodology described in Section 4.4. The obtained semi-purified, sBC was later used for comparison of cofactor efficiency.

\subsection{Pigment Semi-Purification}

ACN semi-purification was performed using SPE following the methodology described by Rodriguez-Saona and Wrolstad [46] with minor modifications. Briefly, crude ACN extracts were diluted in acidified water prior to SPE using Waters Sep-pak C18 cartridges (Waters, Milford, MA, USA). Cartridges were activated with methanol and then washed with $0.01 \% \mathrm{HCl}(v / v)$ acidified water, after which the crude extract was applied to the cartridge. Salts, sugars, and organic acids were removed using two volumes of 
acidified water followed by washing with three to four volumes of ethyl acetate to remove less polar phenolics. Semi-purified pigments were eluted from the column using 0.01\% $\mathrm{HCl}(v / v)$ acidified methanol. The solvent was then evaporated using a rotavapor at $45^{\circ} \mathrm{C}$, and pigments were resolubilized in acidified water and stored under refrigeration until further use.

\subsection{Fractionation of Different Anthocyanins}

Fractions of different ACNs from semi-purified Berberis boliviana extract prepared in Section 4.4 were obtained using semi-preparative HPLC-PDA (Shimadzu, Columbia, MD, USA). Reverse phase chromatographic separation was achieved using a Synergi $4 \mu \mathrm{m}$ Max-RP $80 \AA$ column of $250 \times 21.2 \mathrm{~mm}$ dimensions (Phenomenex, Torrance, CA, USA) and a binary solvent system composed of A: $4.5 \%$ formic acid in water $(v / v)$ and B: acetonitrile at a flow rate of $10 \mathrm{~mL} / \mathrm{min}$. Elution gradient started at $10-20 \%$ B from 0 to $20 \mathrm{~min}, 20-40 \%$ B from 20 to $21 \mathrm{~min}$, and $40 \%$ B from 21 to $24 \mathrm{~min}$. Collected fractions were concentrated using SPE to remove formic acid and acetonitrile, and residual methanol was evaporated using a rotavapor at $45^{\circ} \mathrm{C}$. Isolated fractions were resolubilized with acidified water and stored under refrigeration until further use.

\subsection{Anthocyanin and Pyranoanthoycanin Identification}

ACN and PACN tentative identification were carried out using a Nexera-i-LC-2040 3D ultra-high performance liquid chromatograph (Shimadzu, Columbia, MD, USA) coupled with an LCMS-8040 triple quadrupole mass spectrometer with an ESI interface (Shimadzu, Columbia, MD, USA). Chromatographic separation was achieved using a Synergi $4 \mu \mathrm{m}$ Max RP-80 A $250 \times 4.6 \mathrm{~mm}$ column (Phenomenex, Torrance, CA, USA) and a binary solvent system consisting of A: $4.5 \%$ formic acid and B: acetonitrile with a gradient of $5-45 \%$ B from 0 to $20 \mathrm{~min}$ and $45 \%$ B from 20 to $25 \mathrm{~min}$. After chromatographic separation, a volume of approximately $0.2 \mathrm{~mL} / \mathrm{min}$ was diverted into the MS/MS for analyses. Tentative identification of pigments was carried out based on their elution time, UV-Vis spectral characteristics, and corresponding $m / z$ using ESI-MS/MS. Mass spectrometry analyses were conducted under positive ion mode with $1.5 \mathrm{~L} / \mathrm{min}$ nebulizing gas flow, $15 \mathrm{~L} / \mathrm{min}$ drying gas, a desolvation gas temperature of $230^{\circ} \mathrm{C}$, and collision energy of $-35 \mathrm{eV}$. Spectral data were acquired using total ion scan mode from $m / z 100$ to 1000 and precursor ion scan mode for the most common six anthocyanidins in nature $(271,287,301,303,317$, and $331 \mathrm{~m} / \mathrm{z}$ ). Identification of new colored compounds was carried out using precursor ion scans with the expected PACN aglycone $m / z$ listed in the tables in Figures 1, 3 and 5 for each experimental section. Data analysis and interpretation were performed using Lab Solutions Software Ver. 1 (Shimadzu, Columbia, MD, USA).

\subsection{Monomeric Anthocyanin Quantitation}

Monomeric ACNs were quantified using the $\mathrm{pH}$ differential method [48]. Briefly, semi-purified ACN extracts or isolated ACN fractions were diluted in $0.025 \mathrm{M}$ potassium chloride buffer at $\mathrm{pH} 1$ or $0.4 \mathrm{M}$ sodium acetate buffer at $\mathrm{pH} 4.5$. The difference of absorbance at their respective $\lambda_{\text {vis-max }}$ and $700 \mathrm{~nm}$ was measured using a SpectraMax M2 plate reader (Molecular Devices, Sunnyvale, CA, USA). The monomeric ACN content in SBC was expressed as cyanidin-3-O-glucoside equivalents while the content in each ACN fraction from Berberis boliviana was expressed in equivalents of its most abundant ACN (delphinidin-3-O-glucoside, cyanidin-3-O-glucoside, petunidin-3-O-glucoside, or malvidin-3-O-glucoside) using the corresponding molecular weight and molar absorptivities reported in the literature [48,49].

\subsection{Pyranoanthocyanin Formation-Comparing Different Types of Cofactors}

PACN formation was carried out following the methodology described by Straathof and Giusti [29] with minor modifications. Briefly, stock solutions of sBC and three different types of cofactors (PA, acetone, and $\mathrm{CA}$ ) were prepared using $\mathrm{pH} 3.1$ acidified water 
containing $0.1 \%$ potassium sorbate $(w / v)$ and $0.1 \%$ sodium benzoate $(w / v)$. Stock solutions of pigment and cofactor were mixed and diluted to a pigment concentration of $80 \mu \mathrm{M}$ and a cofactor concentration of $2.4 \mathrm{mM}$ (1:30 pigment to cofactor molar ratio). The final solution was adjusted to $\mathrm{pH} 3.1 \pm 0.05$ with $1 \mathrm{M} \mathrm{NaOH}$ and $2 \mathrm{~N} \mathrm{HCl}$ when needed. Samples were placed into HPLC vials and stored in the dark in a Roto-Therm ${ }^{\mathrm{TM}} \mathrm{H} 2020$ benchtop incubator (Benchmark Scientific, Edison, NJ, USA) set at $45^{\circ} \mathrm{C}$. Every $24 \mathrm{~h}$, the vial was removed, run on the HPLC, and returned to the incubator for additional incubation, up to $72 \mathrm{~h}$ total.

\subsection{Pyranoanthocyanin Formation-Comparing Different Hydroxycinnamic Acids}

The effects of minor structural differences among different hydroxycinnamic acids on PACN formation were evaluated following the methodology described in Section 4.8. The sBC extract was used as the pigment source, and four different hydroxycinnamic acids (CA, pCA, FA, and SA) were tested as cofactors at equivalent 1:30 ACN to cofactor molar ratios and incubated at $45{ }^{\circ} \mathrm{C}$ in $\mathrm{pH} 3.1$ solution in the dark.

\subsection{Pyranoanthocyanin Formation-Comparing Different Aglycones}

The effect of the aglycone structure on the formation efficiency of PACNs was evaluated using ACN-rich fractions containing a different aglycone and the cofactor identified as producing the highest PACN yield (in this case, CA) following the methodology described in Section 4.8. Berberis boliviana was selected as the pigment source because it contains four of the six most common ACNs found in nature in relatively similar proportions with glycosylations (either glucose or rutinose) only on position C3 [32]. CA was used as a cofactor at a 1:30 ACN to cofactor molar ratio, and samples were incubated at $45^{\circ} \mathrm{C}$ in pH 3.1 solution in the dark.

\subsection{Monitoring Pyranoanthocyanin Formation and Anthocyanin Changes Over Time}

Total pigment content and PACN formation were monitored during incubation using HPLC-PDA (Shimadzu, Columbia, MD, USA) and calculated using the areas under the curve (AUC) of the compounds of interest. The system consisted of two LC-20AD pumps, a CBM-20A controller, a SIL-20AC refrigerated autosampler, an SPD-M20A PDA detector, and a CTO-20A column oven. Chromatographic separation was achieved using the same column, solvents, and gradient parameters as in Section 4.6.

PACN formation yield was calculated using Equation (1):

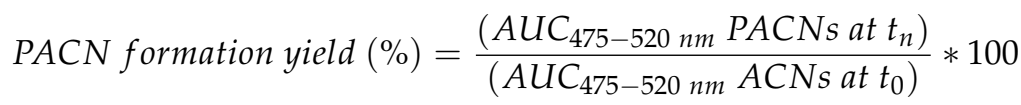

The percent of pigment remaining was calculated using Equation (2):

$$
\text { Percent of Pigment Remaining }=\frac{\left(A U C_{475-520 ~ n m} A C N s+P A C N s \text { at } t_{n}\right)}{\left(A U C_{475-520 ~ n m} A C N s \text { at } t_{0}\right)} * 100
$$

The content of PACNs, as the percentage of total pigment at a given time point, was calculated using Equation (3):

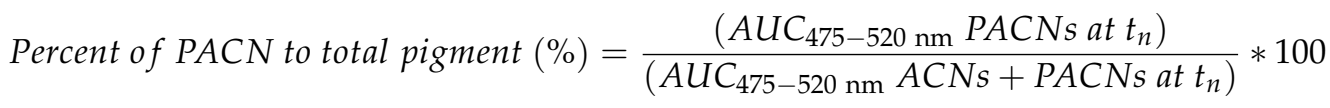

\subsection{Statistical Evaluation of Data}

Data from each replication were collected from duplicate samples and experiments were conducted in triplicate. Data were expressed as mean \pm standard error of means. Differences among treatments were analyzed using a one-way analysis of variance (ANOVA) with Bonferroni post-hoc tests. Analyses were conducted using GraphPad Prism (GraphPad Software Inc., La Jolla, CA, USA). A p-value lower than 0.05 was considered significant. 


\section{Conclusions}

The chemical structure of the cofactor and ACN type impacted PACN yields with significant differences detected after only $24 \mathrm{~h}$ of incubation. CA and FA were the most efficient cofactors with similar PACN yields when using cyanidin-glycosides from $\mathrm{sBC}$ as the ACN source $(\sim 19 \%$ and $\sim 14 \%$, respectively). Among the different types of aglycones tested, malvidin-glycosides were the most efficient for PACN formation with CA $(\sim 20 \%$ after $24 \mathrm{~h}$ ). Overall, this research demonstrated that cyanidin- or malvidin-derived ACNs in combination with CA or FA can produce high amounts of PACNs under accelerated formation conditions. These results highlight the importance of the ACN source and cofactor selection for the efficient production of PACNs. Given their scarcity in nature, this efficient production could facilitate their use by the industry as naturally derived colorants with increased stability.

Author Contributions: Conceptualization, G.M.-C. and M.M.G.; methodology, G.M.-C. and D.M.V.; validation, G.M.-C. and D.M.V.; formal analysis, G.M.-C. and D.M.V.; investigation, G.M.-C. and D.M.V.; resources, M.M.G.; data curation, G.M.-C. and D.M.V.; writing-original draft preparation, G.M.-C. and D.M.V.; writing-review and editing, G.M.-C., D.M.V. and M.M.G.; visualization, G.M.C., D.M.V. and M.M.G.; supervision, M.M.G.; project administration, G.M.-C. and M.M.G.; funding acquisition, M.M.G. All authors have read and agreed to the published version of the manuscript.

Funding: This research was supported in part by FONDECYT-CONCYTEC, grant number 225-2015FONDECYT and the USDA National Institute of Food and Agriculture, Hatch Project OHO01423, Accession Number 1014136.

Institutional Review Board Statement: Not applicable.

Informed Consent Statement: Not applicable.

Data Availability Statement: The data that supports the findings of this study are available from the corresponding author upon reasonable request.

Acknowledgments: The authors would like to thank Yesen Cheng for his technical assistance in this project. The authors would also like to thank Molly J. Davis for editing the manuscript.

Conflicts of Interest: The authors declare no conflict of interest.

\section{References}

1. Mazza, G.; Brouillard, R. Recent Developments in the Stabilization of Anthocyanins in Food Products. Food Chem. 1987, 25, 207-225. [CrossRef]

2. Sigurdson, G.T.; Tang, P.; Giusti, M.M. Natural Colorants: Food Colorants from Natural Sources. Annu. Rev. Food Sci. Technol. 2017, 8, 261-280. [CrossRef] [PubMed]

3. Bateman, B.; Warner, J.O.; Hutchinson, E.; Dean, T.; Rowlandson, P.; Gant, C.; Grundy, J.; Fitzgerald, C.; Stevenson, J. The Effects of a Double Blind, Placebo Controlled, Artificial Food Colourings and Benzoate Preservative Challenge on Hyperactivity in a General Population Sample of Preschool Children. Arch. Dis. Child. 2004, 89, 506-511. [CrossRef] [PubMed]

4. McCann, D.; Barrett, A.; Cooper, A.; Crumpler, D.; Dalen, L.; Grimshaw, K.; Kitchin, E.; Lok, K.; Porteous, L.; Prince, E.; et al. Food Additives and Hyperactive Behaviour in 3-Year-Old and 8/9-Year-Old Children in the Community: A Randomised, Double-Blinded, Placebo-Controlled Trial. Lancet 2007, 370, 1560-1567. [CrossRef]

5. He, J.; Monica Giusti, M. Anthocyanins: Natural Colorants with Health-Promoting Properties. Annu. Rev. Food Sci. Technol. 2010, 1, 163-187. [CrossRef]

6. Farr, J.E.; Giusti, M.M. Investigating the Interaction of Ascorbic Acid with Anthocyanins and Pyranoanthocyanins. Molecules 2018, 23, 744. [CrossRef] [PubMed]

7. Pangestu, N.P.; Miyagusuku-Cruzado, G.; Giusti, M.M. Copigmentation with Chlorogenic and Ferulic Acid Affected Color and Anthocyanin Stability in Model Beverages Colored with Sambucus Peruviana, Sambucus Nigra and Daucus Carota during Storage. Foods 2020, 9, 1476. [CrossRef] [PubMed]

8. Miyagusuku-Cruzado, G.; Jiménez-Flores, R.; Giusti, M.M. Whey Protein Addition and Its Increased Light Absorption and Tinctorial Strength of Model Solutions Colored with Anthocyanins. J. Dairy Sci. 2020, 104, 6449-6462. [CrossRef]

9. Weber, F.; Boch, K.; Schieber, A. Influence of Copigmentation on the Stability of Spray Dried Anthocyanins from Blackberry. LWT Food Sci. Technol. 2017, 75, 72-77. [CrossRef]

10. Tang, P.; Giusti, M.M. Metal Chelates of Petunidin Derivatives Exhibit Enhanced Color and Stability. Foods 2020, 9, 1426. [CrossRef] 
11. Sun, J.; Li, X.; Luo, H.; Ding, L.; Jiang, X.; Li, X.; Jiao, R.; Bai, W. Comparative Study on the Stability and Antioxidant Activity of Six Pyranoanthocyanins Based on Malvidin-3-Glucoside. J. Agric. Food Chem. 2020, 68, 2783-2794. [CrossRef]

12. Farr, J.E.; Sigurdson, G.T.; Giusti, M.M. Influence of Cyanidin Glycosylation Patterns on Carboxypyranoanthocyanin Formation. Food Chem. 2018, 259, 261-269. [CrossRef] [PubMed]

13. Velenosi, M.; Crupi, P.; Perniola, R.; Marsico, A.D.; Salerno, A.; Alexandre, H.; Archidiacono, N.; Ventura, M.; Cardone, M.F. Color Stabilization of Apulian Red Wines through the Sequential Inoculation of Starmerella Bacillaris and Saccharomyces Cerevisiae. Molecules 2021, 26, 907. [CrossRef] [PubMed]

14. Marquez, A.; Serratosa, M.P.; Merida, J. Pyranoanthocyanin Derived Pigments in Wine: Structure and Formation during Winemaking. J. Chem. 2013, 2013, 713028. [CrossRef]

15. Bakker, J.; Timberlake, C.F. Isolation, Identification, and Characterization of New Color-Stable Anthocyanins Occurring in Some Red Wines. J. Agric. Food Chem. 1997, 45, 35-43. [CrossRef]

16. Mateus, N.; Silva, A.M.S.; Vercauteren, J.; de Freitas, V. Occurrence of Anthocyanin-Derived Pigments in Red Wines. J. Agric. Food Chem. 2001, 49, 4836-4840. [CrossRef]

17. He, J.; Carvalho, A.R.F.; Mateus, N.; de Freitas, V. Spectral Features and Stability of Oligomeric Pyranoanthocyanin-Flavanol Pigments Isolated from Red Wines. J. Agric. Food Chem. 2010, 58, 9249-9258. [CrossRef] [PubMed]

18. de Freitas, V.; Mateus, N. Formation of Pyranoanthocyanins in Red Wines: A New and Diverse Class of Anthocyanin Derivatives. Anal. Bioanal. Chem. 2011, 401, 1467-1477. [CrossRef]

19. Schwarz, M.; Wray, V.; Winterhalter, P. Isolation and Identification of Novel Pyranoanthocyanins from Black Carrot (Daucus Carota L.) Juice. J. Agric. Food Chem. 2004, 52, 5095-5101. [CrossRef]

20. Blanco-Vega, D.; López-Bellido, F.J.; Alía-Robledo, J.M.; Hermosín-Gutiérrez, I. HPLC-DAD-ESI-MS/MS Characterization of Pyranoanthocyanins Pigments Formed in Model Wine. J. Agric. Food Chem. 2011, 59, 9523-9531. [CrossRef]

21. Fossen, T.; Andersen, Ø.M. Anthocyanins from Red Onion, Allium Cepa, with Novel Aglycone. Phytochemistry 2003, 62, 1217-1220. [CrossRef]

22. Andersen, Ø.M.; Fossen, T.; Torskangerpoll, K.; Fossen, A.; Hauge, U. Anthocyanin from Strawberry (Fragaria ananassa) with the Novel Aglycone, 5-Carboxypyranopelargonidin. Phytochemistry 2004, 65, 405-410. [CrossRef]

23. Peng, Y.; Zhang, H.; Liu, R.; Mine, Y.; McCallum, J.; Kirby, C.; Tsao, R. Antioxidant and Anti-Inflammatory Activities of Pyranoanthocyanins and Other Polyphenols from Staghorn Sumac (Rhus Hirta L.) in Caco-2 Cell Models. J. Funct. Foods 2016, 20, 139-147. [CrossRef]

24. Liu, S.; Laaksonen, O.; Yang, W.; Zhang, B.; Yang, B. Pyranoanthocyanins in Bilberry (Vaccinium Myrtillus L.) Wines Fermented with Schizosaccharomyces Pombe and Their Evolution during Aging. Food Chem. 2020, 305, 125438. [CrossRef]

25. Rein, M.J.; Ollilainen, V.; Vahermo, M.; Yli-Kauhaluoma, J.; Heinonen, M. Identification of Novel Pyranoanthocyanins in Berry Juices. Eur. Food Res. Technol. 2005, 220, 239-244. [CrossRef]

26. Mateus, N.; Oliveira, J.; Haettich-Motta, M.; de Freitas, V. New Family of Bluish Pyranoanthocyanins. J. Biomed. Biotechnol. 2004, 2004, 299-305. [CrossRef] [PubMed]

27. Zhu, X.; Giusti, M.M. Pyranoanthocyanin Formation Rates and Yields as Affected by Cyanidin-3-Substitutions and Pyruvic or Caffeic Acids. Food Chem. 2021, 345, 128776. [CrossRef] [PubMed]

28. Hoehn, M.E. Altering pH, Temperature and Cofactors to Increase the Formation of the More Stable Anthocyanin Derived Pyranoanthocyanin. Master's Thesis, The Ohio State University, Columbus, OH, USA, 2019.

29. Straathof, N.; Giusti, M.M. Improvement of Naturally Derived Food Colorant Performance with Efficient Pyranoanthocyanin Formation from Sambucus Nigra Anthocyanins Using Caffeic Acid and Heat. Molecules 2020, 25, 5998. [CrossRef]

30. Oliveira, J.; de Freitas, V.; Silva, A.M.S.; Mateus, N. Reaction between Hydroxycinnamic Acids and Anthocyanin-Pyruvic Acid Adducts Yielding New Portisins. J. Agric. Food Chem. 2007, 55, 6349-6356. [CrossRef] [PubMed]

31. Schwarz, M.; Wabnitz, T.C.; Winterhalter, P. Pathway Leading to the Formation of Anthocyanin-Vinylphenol Adducts and Related Pigments in Red Wines. J. Agric. Food Chem. 2003, 51, 3682-3687. [CrossRef] [PubMed]

32. del Carpio Jiménez, C.; Serrano Flores, C.; He, J.; Tian, Q.; Schwartz, S.J.; Giusti, M.M. Characterisation and Preliminary Bioactivity Determination of Berberis Boliviana Lechler Fruit Anthocyanins. Food Chem. 2011, 128, 717-724. [CrossRef]

33. Topić Božič, J.; Ćurko, N.; Kovačević Ganić, K.; Butinar, L.; Albreht, A.; Vovk, I.; Korte, D.; Mozetič Vodopivec, B. Synthesis of Pyranoanthocyanins from Pinot Noir Grape Skin Extract Using Fermentation with High Pyranoanthocyanin Producing Yeasts and Model Wine Storage as Potential Approaches in the Production of Stable Natural Food Colorants. Eur. Food Res. Technol. 2020, 246, 1141-1152. [CrossRef]

34. Eiro, M.J.; Heinonen, M. Anthocyanin Color Behavior and Stability during Storage: Effect of Intermolecular Copigmentation. J. Agric. Food Chem. 2002, 50, 7461-7466. [CrossRef] [PubMed]

35. Fan, L.; Wang, Y.; Xie, P.; Zhang, L.; Li, Y.; Zhou, J. Copigmentation Effects of Phenolics on Color Enhancement and Stability of Blackberry Wine Residue Anthocyanins: Chromaticity, Kinetics and Structural Simulation. Food Chem. 2019, 275, 299-308. [CrossRef] [PubMed]

36. Boulton, R. The Copigmentation of Anthocyanins and Its Role in the Color of Red Wine: A Critical Review. Am. J. Enol. Vitic. 2001, 52, 67-87.

37. Zheng, W.; Wang, S.Y. Oxygen Radical Absorbing Capacity of Phenolics in Blueberries, Cranberries, Chokeberries, and Lingonberries. J. Agric. Food Chem. 2003, 51, 502-509. [CrossRef] 
38. Frond, A.D.; Iuhas, C.I.; Stirbu, I.; Leopold, L.; Socaci, S.; Andreea, S.; Ayvaz, H.; Andreea, S.; Mihai, S.; Diaconeasa, Z.; et al. Phytochemical Characterization of Five Edible Purple-Reddish Vegetables: Anthocyanins, Flavonoids, and Phenolic Acid Derivatives. Molecules 2019, 24, 1536. [CrossRef] [PubMed]

39. Torre, P.; Aliakbarian, B.; Rivas, B.; Domínguez, J.M.; Converti, A. Release of Ferulic Acid from Corn Cobs by Alkaline Hydrolysis. Biochem. Eng. J. 2008, 40, 500-506. [CrossRef]

40. Schwarz, M.; Winterhalter, P. A Novel Synthetic Route to Substituted Pyranoanthocyanins with Unique Colour Properties. Tetrahedron Lett. 2003, 44, 7583-7587. [CrossRef]

41. Gaspar, A.; Garrido, E.M.; Esteves, M.; Quezada, E.; Milhazes, N.; Garrido, J.; Borges, F. New Insights into the Antioxidant Activity of Hydroxycinnamic Acids: Synthesis and Physicochemical Characterization of Novel Halogenated Derivatives. Eur. J. Med. Chem. 2009, 44, 2092-2099. [CrossRef]

42. Song, B.J.; Sapper, T.N.; Burtch, C.E.; Brimmer, K.; Goldschmidt, M.; Ferruzzi, M.G. Photo- and Thermodegradation of Anthocyanins from Grape and Purple Sweet Potato in Model Beverage Systems. J. Agric. Food Chem. 2013, 61, 1364-1372. [CrossRef]

43. Wang, F.; Li, H.; Qin, Y.; Mao, Y.; Zhang, B.; Deng, Z. Effects of Heat, Ultrasound, and Microwave Processing on the Stability and Antioxidant Activity of Delphinidin and Petunidin. J. Food Biochem. 2019, 43, e12818. [CrossRef] [PubMed]

44. Benito, S.; Morata, A.; Palomero, F.; González, M.C.; Suárez-Lepe, J.A. Formation of Vinylphenolic Pyranoanthocyanins by Saccharomyces Cerevisiae and Pichia Guillermondii in Red Wines Produced Following Different Fermentation Strategies. Food Chem. 2011, 124, 15-23. [CrossRef]

45. Hillebrand, S.; Schwarz, M.; Winterhalter, P. Characterization of Anthocyanins and Pyranoanthocyanins from Blood Orange [Citrus Sinensis (L.) Osbeck] Juice. J. Agric. Food Chem. 2004, 52, 7331-7338. [CrossRef] [PubMed]

46. Rodríguez-Saona, L.E.; Wrolstad, R.E. Extraction, Isolation, and Purifification of Anthocyanins. Curr. Protoc. Food Anal. Chem. 2001, F1.1.1-F1.1.11. [CrossRef]

47. Giusti, M.M.; Wrolstad, R.E. Characterization of Red Radish Anthocyanins. J. Food Sci. 1996, 61, 322-326. [CrossRef]

48. Giusti, M.M.; Wrolstad, R.E. Characterization and Measurement of Anthocyanins by UV-Visible Spectroscopy. Curr. Protoc. Food Anal. Chem. 2001, 1-13. [CrossRef]

49. Cabrita, L.; Fossen, T.; Andersen, Ø.M. Colour and Stability of the Six Common Anthocyanidin 3-Glucosides in Aqueous Solutions. Food Chem. 2000, 68, 101-107. [CrossRef] 